treatment" (2 trials) in patients with either "acute" (4 trials), "chronic" (8 trials), or "minimal" (3 trials) HE or else for the "prevention of HE recurrence" (2 trials). Most trials were of adequate quality; the total sample size was 1037 patients, but data were not extractable for all outcomes. The relative proportion of patients experiencing clinical improvement with rifaximin, compared to all control regimen, was 1.26 (95\% CI 1.05 to 1.52 , $\mathrm{p}=0.014$ ). Broadly consistent benefits were shown for individual outcome variables for example, asterixis (SMD $-0.32,95 \% \mathrm{CI}-0.62$ to $-0.02, p=0.038,5$ trials); EEG (SMD $-0.49,95 \% \mathrm{CI}-0.92$ to $-0.07, \mathrm{p}=0.023,2$ trials $)$ and blood ammonia $(\mathrm{SMD}=-0.25,95 \% \mathrm{CI}$ -0.48 to $-0.02, p=0.034,8$ trials), although not for mental status (SMD $-0.19,95 \% \mathrm{CI}-0.47$ to $0.10, \mathrm{p}=0.20,5$ trials) or NCTs (SMD $0.05,95 \% \mathrm{CI}-0.19$ to $0.28, \mathrm{p}=0.8,7$ trials). SMDs were stronger in the eight trials in chronic hepatic encephalopathy (asterixis -0.34; EEG -0.56; blood ammonia -0.26; mental state -0.45 ; NCTs 0.36 ). No significant differences were observed in side-effects between rifaximin and control regimens.

Conclusion Rifaximin is an efficacious and safe treatment for HE when compared to a variety of other treatments regimens; the benefits are most marked in patients with chronic HE.

Competing interests None declared.

\section{PTU-049 MODULATION OF TOLL-LIKE RECEPTOR GENES WITH SELECTIVE GUT DECONTAMINATION IN CIRRHOTIC ANIMALS PREVENTS THE DEVELOPMENT OF ACUTE-ON CHRONIC LIVER FAILURE (ACLF)}

doi:10.1136/gutjnl-2012-302514c.49

${ }^{1} \mathrm{~N}$ Shah, ${ }^{*} \mathrm{D}$ Dhar, ${ }^{1} \mathrm{~N}$ A Davies, ${ }^{1} \mathrm{R}$ P Mookerjee, ${ }^{1} \mathrm{R}$ Jalan. ${ }^{1}$ Hepatology, UCL Institute of Hepatology, London, UK; ${ }^{2}$ Surgery, UCL Institute of Hepatology, London, UK

Introduction Acute deterioration in the liver function following precipitating illness in patients with cirrhosis often lead to multiple organ failure. Inflammation is thought to be a major contributing factor. We hypothesised that toll like receptor's (TLRs) play a pivotal role in lipopolysaccharide (LPS) mediated cytokine surge in patients with cirrhosis culminating in acute-on-chronic liver failure (ACLF). Selective gut decontamination with Norfloxacin would dampen this inflammatory cascade.

Methods Global hepatic gene expression was studied in a rodent model of cirrhosis induced by bile duct ligation (BDL) (Agilent microarray) and compared the results to sham animals. TLR related genes were among the differentially expressed genes in the liver. We validated gene expression in the Liver and Kidney in a second set of experiment in Sham, BDL, BDL+LPS, BDL+Norfloxacin, BDL +Norfloxacin+LPS groups using the quantitative real-time PCR. Cytokines (Becton Dickenson) and biochemistry were measured (COBAS Integra 400).

Results Among the several genes with high expression in the BDL group as detected by the microarray, both TLR 4 and 2 had significantly higher expression in liver and Kidney compared with the sham group. We also found a marked upregulation of the C-C motif ligand $2(\mathrm{CCl} 2)$ and $\mathrm{C}-\mathrm{X}-\mathrm{C}$ motif ligand $2(\mathrm{CxCl})$ Furthermore, LPS administration in BDL animals accentuated not only the TLRs (4 and 2) expression in both kidney and liver but also the Cxcl2 and $\mathrm{CCl} 2$ in both these organs associated with deterioration organ function as suggested with a rise in the liver enzyme, creatinine and a rise in plasma and tissue cytokines. Norfloxacin pre-treatment in $\mathrm{BDL}$ group (BDL+Norfloxacin) attenuated the TLR4 and TLR2 expression in both liver and kidney. Selective decontamination with Norfloxacin in BDL +LPS animals limited the upregulation of TLR4 and TLR2 in the Liver and Kidney. It also prevented the upregulation of the CxcL2 and CCl2 in both these organs. This was associated with significant improvement in liver enzymes, creatinine and cytokines.

Conclusion This study shows that the liver and kidneys in cirrhotic animals are primed by upregulation of TLR's and its downstream inflammatory mediators in the Liver and Kidney which make them exquisitely sensitive to the effects of superimposed infection/ inflammation leading to organ failure. Selective decontamination with Norfloxacin prevents the progression of ACLF by reducing gene expression of TLR 2 and 4 and its associated inflammatory adaptors.

Competing interests None declared.

\section{PTU-050 EFFECT OF VITAMIN E AND ALFA LIPOIC ACID (ALA) IN NON-ALCOHOLIC FATTY LIVER DISEASE: A RANDOMISE PLACEBO CONTROL OPEN LABEL PROSPECTIVE CLINICAL TRIAL: V A I N TRIAL}

doi:10.1136/gutjnl-2012-302514c.50

${ }^{1} \mathrm{P}$ Basu, ${ }^{2}{ }^{2} \mathrm{~N}$ James Shah, ${ }^{2} \mathrm{~S}$ Farhat. ${ }^{1}$ Gastroenterolgy and Liver Transplant, Columbia University, College of Physicians and Surgeons, New York, New York, USA; ${ }^{2}$ Internal Medicine, North Shore University, Forest Hills, New York, USA

Introduction Non-Alcoholic Fatty Liver Disease (NAFLD) is a global epidemic. NAFLD progress to Non-Alcoholic Steatohepatitis (NASH), cirrhosis and cancer. Obesity and insulin insensitivity is the hallmark. Liver participates in glucose and fatty acid homeostasis. Nonexpelled free fatty acid and its toxic metabolites impact oxidative stress; free radicals influx which initiate inflammatory cascade to Steatohepatitis (NASH) and activate fibrotic path causing cirrhosis. This clinical trial highlights the effects of anti-oxidants in NAFLD

Methods One hundred and fifty-five $(n=155)$ with BMI over $28 \%$ with NAFLD and NASH were recruited and randomised into Group A ( $n=35)$ - Control, Group B ( $n=40)$ ALA 300 mg, Group C $(n=40)$ Vitamin E 700 IU and Group D (n=40) ALA plus Vitamin E orally for 6 months. Pre and Post BMI, HOMA, Triglyceride, Haemoglobin A1c, Alanine aminotransferase (ALT), Retinol Binding Protein 4, Tumour Necrosis factor $\alpha$ (TNF $\alpha$ ), Leptin and adiponectin levels were compared. Everyone was allowed $1600 \mathrm{cal} /$ day with modest exercise. Exclusion criteria: Diabetics, BMI $>33 \%$, Alcohol intake $>30 \mathrm{~g} /$ day, Hepatitis B, C, Hypothyroidism, medications including herbs and supplements.

Results

\begin{tabular}{llll}
\hline \% Changes & Group B (ALA) & Group C (Vit E) & Group D (ALA + Vit E) \\
\hline$\delta$ Triglyceride & $34.3 \%$ & $37.5 \%$ & $56.4 \%$ \\
$\delta$ HOMA & $54.3 \%$ & $53.7 \%$ & $67.4 \%$ \\
$\delta$ ALT & $20.8 \%$ & $40.7 \%$ & $44.8 \%$ \\
$\delta$ RBP4 & $46.2 \%$ & $46.5 \%$ & $61.9 \%$ \\
$\delta$ Leptin & $44.8 \%$ & $46.9 \%$ & $58.6 \%$ \\
$\delta$ Adiponectin & $55 \%$ & $47.8 \%$ & $52.6 \%$ \\
$\delta$ TNF- $\alpha$ & $59.3 \%$ & $61.9 \%$ & $82.6 \%$ \\
$\delta$ Steatosis Score & $75 \%$ & $73.2 \%$ & $78.7 \%$ \\
$\delta$ Fibrotic Score & $5.9 \%$ & $-6.7 \%$ & $5.6 \%$ \\
\hline
\end{tabular}

Conclusion Pre and post analysis between ALA plus Vitamin E over placebo in 6 month; TG 43\%, HbA1c 14\%, HOMA 62.8\%, ALT 14.4\%, RBR4 50\%, Leptin23\%, Adiponectin19\% TNF $\alpha$ 70\%, and Steatotic score $70.7 \%$. This clinical trial demonstrates the additive effects of ALA and vitamin E in NAFLD and NASH with significant improvements of inflammatory and steatotic score but no difference in the fibrotic score. Therapeutic application of ALA and Vitamin E should be considered for NAFLD.

Competing interests None declared. 